\title{
Blue Phase LC/Polymer Fresnel Lens Fabricated by Holographics
}

Jian Tan, Yue Song, Ji-Liang Zhu, Shui-Bin Ni, Yi-Jun Wang, Xiao-Yang Sun, Jian-Gang Lu, Bo-Ru Yang, and Han-Ping D. Shieh, Fellow, IEEE

\begin{abstract}
A novel fabrication method using holographic exposure for switchable blue phase (BP) liquid crystal (LC)/polymer Fresnel lens was demonstrated. The Fresnel pattern can be achieved by interfering a planar and a spherical wave fronts with a 532-nm laser. The BPLC/polymer Fresnel lens can thus be fabricated without photo mask and controlled with a simpler driving compared with the conventional LC Fresnel lens.
\end{abstract}

Index Terms-Blue phase liquid crystal, Fresnel lens, holographic optical elements.

\section{INTRODUCTION}

$\mathbf{E}$ LECTRICALLY controllable Fresnel lens is widely used in various fields, such as optical imaging, long-distance optical communication, optoelectronic modulator devices and especially in three-dimensional (3-D) display [1]-[4]. Compared to other methods, the Fresnel type 3-D display with liquid crystal (LC) lens has shown many advantages such as low crosstalk, high transmittance and possibility of using similar fabrication process of conventional LC displays. Due to the great application potential, Fresnel type LC lens has attracted much attention [5]-[7] in recent years. However, slow response time and polarization-dependence remain the key issues hindering it from wider application both in communication and display. Blue phase liquid crystal (BPLC) shows many interesting features, including sub-millisecond response time, alignment free and optical isotropic status without electrical field for the applications in field sequential display, phase modulator and 3-D tunable photonic crystals [8]-[11]. Therefore, BPLC is one of the solutions to improve the performance of the Fresnel lens. Also, several kinds of LC lens using BPLC which offers polarization independency but small birefringence have been reported [12]-[14].

Manuscript received August 22, 2013; revised October 09, 2013; accepted November 06, 2013. Date of publication November 14, 2013; date of current version February 10, 2014. This work was supported in part by the 973 Program 2013CB328804 and the National Science Foundation of China under Grant 61275026. The work of H.-P. D. Shieh, J.-G. Lu, and B.-R. Yang was supported by China Star Optoelectronics Technology (CSOT).

J. Tan and J.-G. Lu are with the Department of Electronic Engineering and National Engineering Laboratory for TFT-LCD Materials and Technologies, Shanghai Jiao Tong University, Shanghai 200240, China (e-mail: lujg@sjtu. edu.cn).

Y. Song, J.-L. Zhu, S.-B. Ni, Y.-J. Wang, X.-Y. Sun, and B.-R. Yang are with the Department of Electronic Engineering, Shanghai Jiao Tong University, Shanghai 200240, China.

H.-P. D. Shieh is with the Department of Photonics and Display Institute, National Chiao Tung University, Hsinchu 300, Taiwan.

Color versions of one or more of the figures are available online at http:// ieeexplore.ieee.org.

Digital Object Identifier 10.1109/JDT.2013.2291068

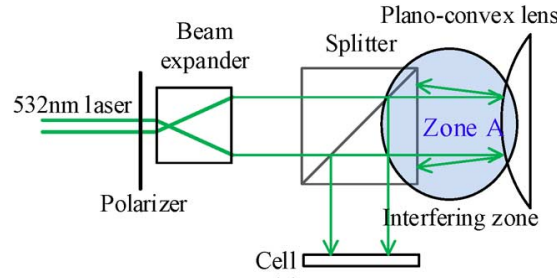

(a)

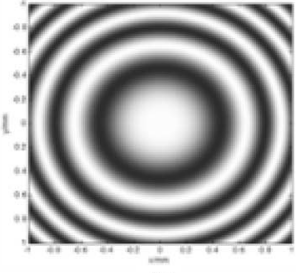

(b)
Fig. 1. (a) Schematics of experimental set-up, and (b) intensity distribution of interfering patterns.

A novel holographic fabrication for Fresnel type BPLC/ polymer lens is presented in this paper. Compared with the multi-electrode driving BPLC lens with the traditional fabrication [15]-[17], this newly proposed Fresnel type BPLC/polymer lens can be controlled in a simpler driving, and its cost can be potentially lowered by using the holographic fabrications.

\section{Design AND FABrication Method}

A Fresnel type interfering intensity distribution was obtained by a planar wave-front and a spherical wave-front. The theory of interfering has been well documented [18]. As shown in Fig. 1(a), the light from the $532 \mathrm{~nm}$ laser in $50 \mathrm{~mW}$ passed through a beam expander and a beam splitter successively, and then was reflected by both of the planar and curved of a plano-convex lens whose focal length is $15 \mathrm{~cm}$. The light reflected from curved and planar surfaces can be considered as spherical and planar wave-fronts, respectively. These two reflected wave-fronts interfered in zone A. Assuming that the light intensities of these two wave-fronts are equal and that their wavelength is $532 \mathrm{~nm}$, the calculated light intensity distribution of interfering pattern at the distance of $40 \mathrm{~cm}$, which was recorded by the receiver, is shown in Fig. 1(b). The bright and dark parts represent high and low intensity, respectively. The focal length of such lens can be calculated by formula $f=r_{1}^{2} / \lambda$, where $r_{1}$ is the radius of the inner-most dark ring and $\lambda$ is wavelength of incident light.

As the reflection changed the polarization direction of light, the light with different polarization direction from plano-convex lens would be reflected by splitter, and then irradiated onto the cell. After the mixture of monomer and LC being irradiated by the Fresnel type interfering pattern, the different extent of polymerization and the distribution of refractive index would correlate with the interfering pattern, as the dosage of exposure was different across the interfering pattern. This polymerized pattern thus can be used as the converging lens. 


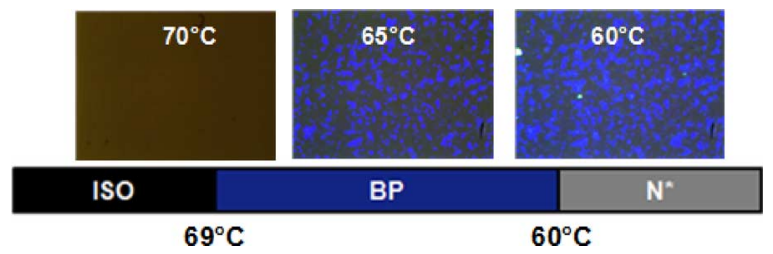

Fig. 2. Morphologies of BPLC observed by a reflective polarizing optical microscopy.

With the BPLC, the refractive index distribution of the lens can be controlled by the electric field which induces the birefringence of BPLC according to the extended Kerr model [20]. The BPLC is optically isotropic when no voltage is applied, the isotropic refractive index can be calculated by:

$$
n_{i} \approx \frac{\left(2 n_{o}+n_{e}\right)}{3},
$$

where $n_{o}$ is ordinary refractive index of LC molecule and $n_{e}$ is extraordinary refractive index. Based on the Extended Kerr model, the birefringence induced by the electric field can be calculated by:

$$
\Delta n_{i n d}(E)=\Delta n_{s}\left[1-\exp \left(-\left(\frac{E}{E_{s}}\right)^{2}\right)\right],
$$

where $\Delta n_{s}$ is saturated birefringence and $E_{s}$ is saturated electric field. For a normal incident light, the refractive index of LC layer is shown in (3):

$$
n(E) \approx n_{i}-\Delta n_{\text {ind }}(E) / 3 .
$$

From (1)to (3), it can be found that a larger applied electric field is needed to induce the birefringence of BPLC and the effective refractive index difference of BPLC is less than that of the conventional nematic LC.

\section{RESULTS \& DISCUSSION}

In the holographic-fabrication method, the Fresnel type patterning was the first step. The substances used in the experiment consisted of $68.0 \mathrm{wt} \%$ of conventional nematic LC (HCCH-03, no $=1.521$ and ne $=1.746$, Jiangsu Hecheng Display Technology Co., Ltd. (HCCH)), 19.9 wt\% of monomer (TMPTA), $8.6 \mathrm{wt} \%$ of crosslinking monomer (NVP), $2.5 \mathrm{wt} \%$ of surfactant (S-271, POE sorbitan monooleate), $0.4 \mathrm{wt} \%$ of photoinitiator (RB), and $0.6 \mathrm{wt} \%$ of co-initiator (NPG). The mixture was filled between two parallel ITO glass substrates by capillary effect, and the cell gap was about $8 \mu \mathrm{m}$. After 30 minutes of exposure in the interfering pattern of $532 \mathrm{~nm}$ laser, the Fresnel pattern was formed.

In the second step, the cell was placed in acetone for 48 hours to wash out the LC molecules, un-polymerized reactive-mesogen, and photoinitiator [23]. Then, the cell was baked at $40^{\circ} \mathrm{C}$ for 24 hours to remove the remaining acetone.

The BPLC mixture (92 $\mathrm{wt} \%$ of HCCH-BP4 and $8 \mathrm{wt} \%$ of ISO(60BA)2, HCCH) was then refilled into the cell in the third step. As shown in Fig. 2, the phase sequence of the mixture

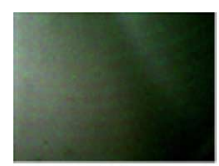

(a)

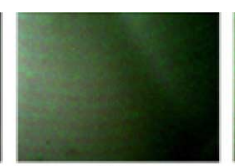

(b)

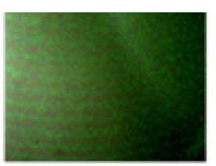

(c)

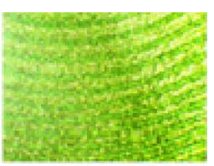

(d)

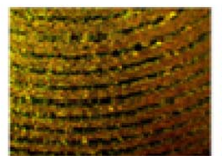

(e)

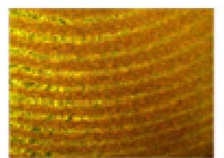

(f)

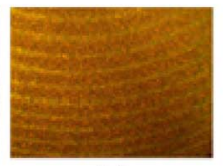

(g)
Fig. 3. Morphologies of refilled BPLC observed by a transmissive polarizing optical microscopy (observing at $65^{\circ} \mathrm{C}$ ) with applied voltage of (a) $0 \mathrm{~V}$, (b) 30 $\mathrm{V}$, (c) $45 \mathrm{~V}$, (d) $50 \mathrm{~V}$, (e) $55 \mathrm{~V}$, (f) $60 \mathrm{~V}$, and (g) $65 \mathrm{~V}$.

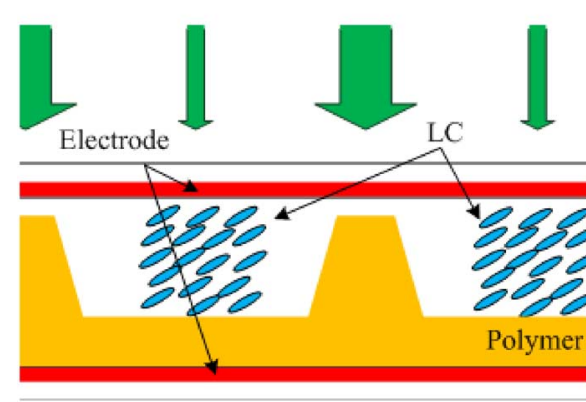

(a)

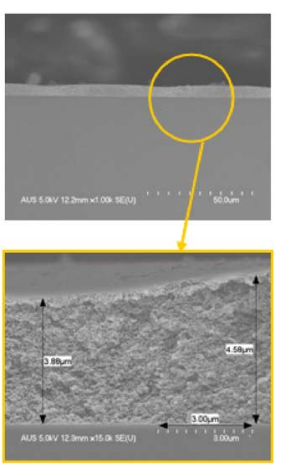

(b)
Fig. 4. Cross-sectional diagram of the LC/polymer lens after holographic fabrication. (a) Schematic diagram and (b) SEM diagram.

shown in Fig. 3 is as follows: Isotropic Phase $-69^{\circ} \mathrm{C}-\mathrm{Blue}$ Phase $-60^{\circ} \mathrm{C}$ - Chiral Nematic Phase

A square wave of $1000 \mathrm{~Hz}$ was applied to the cell at $65^{\circ} \mathrm{C}$. As shown in Fig. 4, when the applied voltage was of less than $45 \mathrm{~V}$, the light spot did not change; however, as the voltage was increased from $45 \mathrm{~V}$ to $53 \mathrm{~V}$, the refractive index difference between LC area and polymer area would increase gradually, and thus the light spot became converged. When the applied voltage was further increased to higher than $53 \mathrm{~V}$, the refractive index difference would degrade due to the electrostriction induced by the strong electric field [24].

Though this method successfully demonstrated the converging capability of BPLC lens, it was observed that the applied voltage for the BPLC/polymer cell was higher than conventional BPLC. As illustrated in Fig. 4 (the SEM diagram), an extra polymer layer was formed between the liquid crystal and electrodes. To avoid this issue, using monomers which can complete the polymerization faster or adopting stronger light sources to expedite the reaction rates are possible solutions.

Another method for visualizing the voltage-dependent focusing power of our BPLC Fresnel lens was made by using a laser of $633 \mathrm{~nm}$ wavelength along with a set of crossed polarizers. The measured focal length was about $20.2 \mathrm{~cm}$, which was close to the calculated focal length, $19.3 \mathrm{~cm}$. As shown in Fig. 5, the focusing capacity could be tuned by applied voltage. When the applied voltage is less than $45 \mathrm{~V}$, the light was not obviously converged. While increasing the voltage gradually 


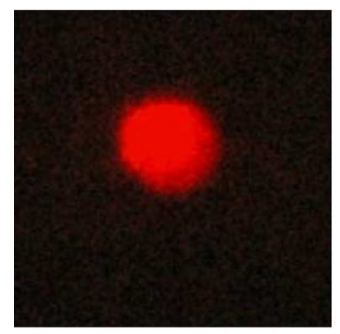

(a)

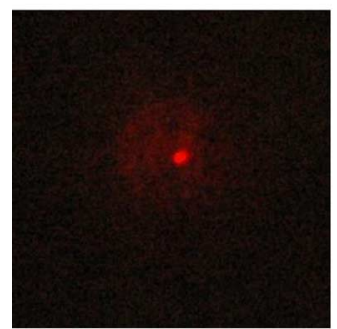

(c)

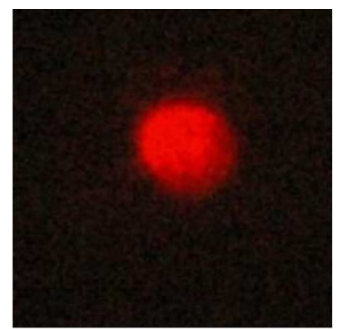

(b)

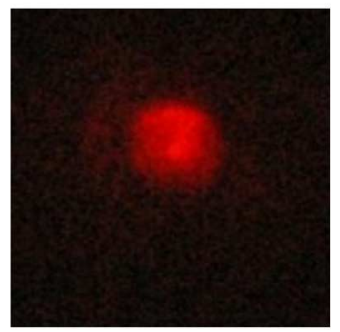

(d)
Fig. 5. The focusing power of refilled BPLC Fresnel lens with applied voltages of (a) $0 \mathrm{~V}$, (b) $45 \mathrm{~V}$, and (c) $53 \mathrm{~V}$ (d) $63 \mathrm{~V}$.

to $53 \mathrm{~V}$, the best convergence occurred. However, if further increasing the voltage to $63 \mathrm{~V}$, as mentioned previously, the de-focusing would occur. In order to show clearly, the exposure time in Fig. 5(a) and 5(c) is extended. Fig. 6 shows the light intensity distribution of focal plane and the simulation result. Fig. 7 shows the measured diffraction efficiency (the red dots) as a function of the applied voltage (the blue dashed line). When the applied voltage on lens was $53 \mathrm{~V}$, the light could be effectively focused and the diffraction efficiency can reach $25 \%$ which is smaller than the simulation result of $40 \%$. The diffraction efficiency can be improved by optimize exposure time according to our previous work [25]. The contrast of converged light intensity and non-converged light intensity was 7.7:1 which is higher than the contrast of the previous work, about 2.4:1 [19]. The formula of focal length, $f=r_{1}^{2} / \lambda$, may explain the wavelength dependence of our lens. With increase of the incident light wavelength, the focal length of lens decreases.

To further demonstrate the fast-switching capability of this BPLC device, we measured the response time. The photodetector was placed at the focal plane of the Fresnel lens. The time measured between the de-focused (10\% of intensity) and focused patterns ( $90 \%$ of intensity) were defined as the focusing and defocusing times, respectively. In order to make a fair as $53 \mathrm{~V}$ and $45 \mathrm{~V}$, respectively, and Von and Voff of nematic LC Fresnel lens were set as $0 \mathrm{~V}$ and $17 \mathrm{~V}$ according to our previous work [25]. The response time comparison of nematic LC Fresnel lens and BPLC Fresnel lens are shown in Fig. 8 where the voltage changed at $3 \mathrm{~ms}$ in focusing and defocusing. Compared with the focusing time, $3.7 \mathrm{~ms}$, and the defocusing time, $3.9 \mathrm{~ms}$, of nematic LC/polymer Fresnel lens, the focusing time, $0.9 \mathrm{~ms}$, and the defocusing time, $1.0 \mathrm{~ms}$, of BPLC/polymer Fresnel lens were much faster, implying that the intrinsic performance of BPLC's fast switching still performs well after refilled. Moreover, by holographic fabrications, the BPLC/polymer Fresnel lenses can be operated with a simpler driving and fabricated with less complicated processes.

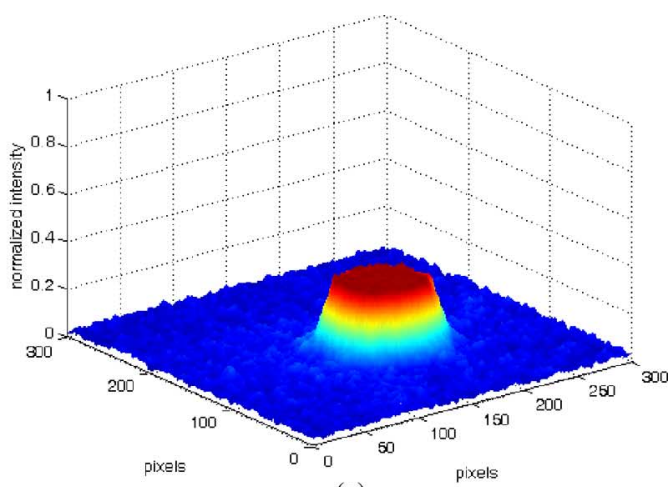

(a)

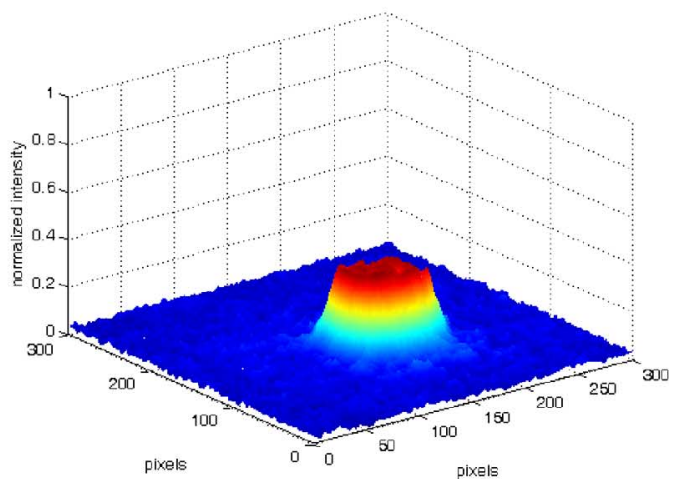

(b)

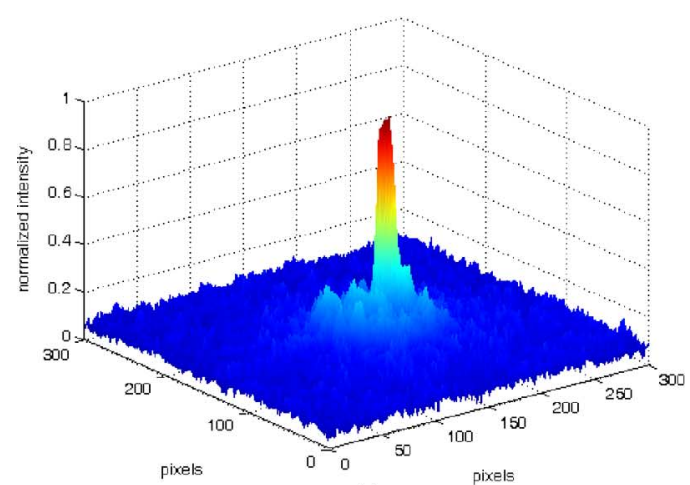

(c)

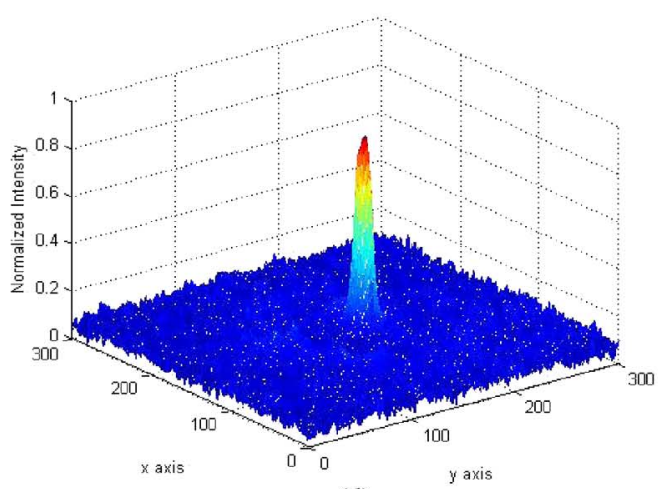

(d)

Fig. 6. The normalized light intensity of focal plane at: (a) $0 \mathrm{~V}$ (b) $45 \mathrm{~V}$ (c) 53 $\mathrm{V}$ and (d) simulation result.

\section{CONClusion}

A novel holographic fabrication method with simple process and mask-free was demonstrated to fabricate BPLC/polymer Fresnel lens. With this method, the BPLC/polymer Fresnel lens 


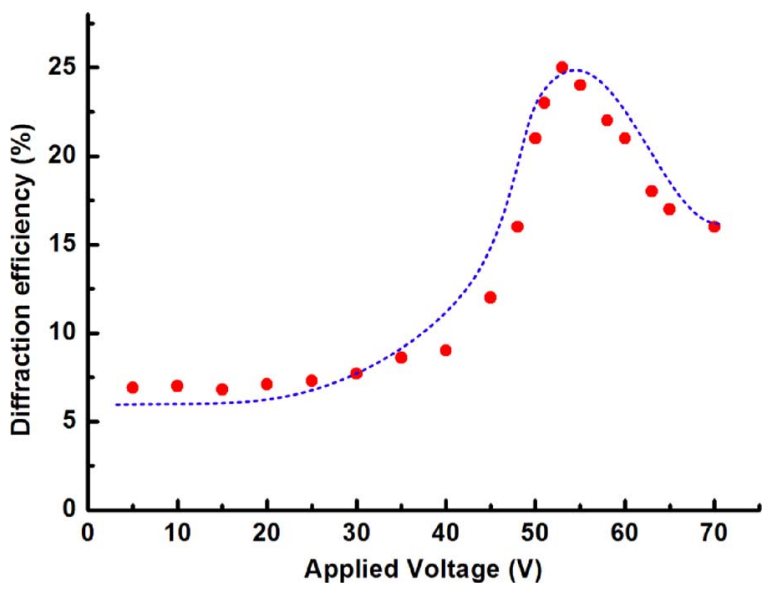

Fig. 7. Measured diffraction efficiency as a function of applied voltage.

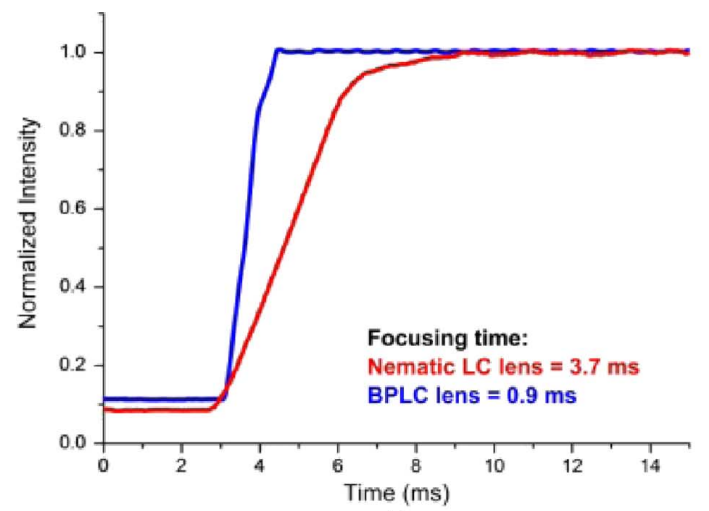

(a)

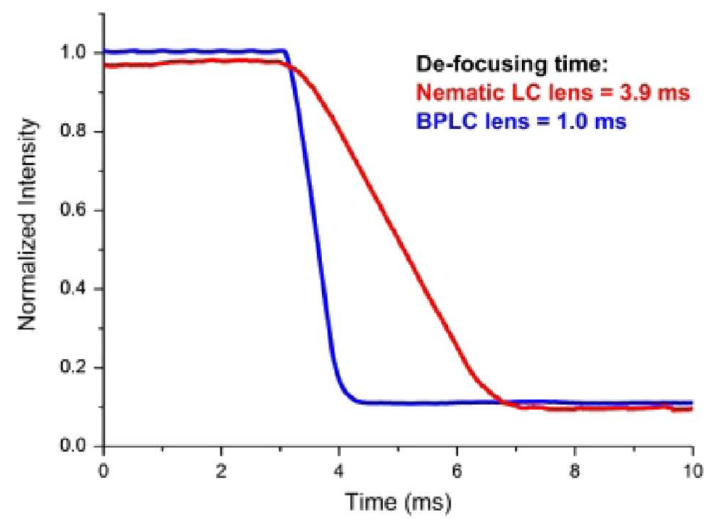

(b)

Fig. 8. The response times of nematic Fresnel lens and BPLC (at $65^{\circ} \mathrm{C}$ ) Fresnel lens: (a) focusing, and (b) de-focusing time.

shows good focusing power, high diffraction efficiency, and fast focusing time of sub-millisecond. Therefore the newly proposed lens has potential for the advanced applications of 3-D display, spectrometer, light modulator and fast-response optical switch.

\section{REFERENCES}

[1] N. Kitatura, S. Ogata, and Y. Mori, "Spectrometer employing a microfresnel lens," Opt. Eng., vol. 34, pp. 584-488, 1995.
[2] M. Ferstl and A. M. Frisch, "Static and dynamic Fresnel zone lenses for optical interconnections," J. Mod. Opt., vol. 43, no. 7, pp. 1451-1462, 2009.

[3] M. Hain, R. Glöckner, S. Bhattacharya, D. Dias, S. Stankovic, and T. Tschudi, "Fast switching liquid crystal lenses for a dual focus digital versatile disc pickup," Opt. Commun., vol. 18, no. 5-6, pp. 291-299, 2001.

[4] J. G. Lu, X. F. Sun, Y. Song, and H. P. D. Shieh, "2-D/3-D switchable display by Fresnel-type LC lens," J. Display Technol., vol. 7, no. 4, pp. 215-219, Nov. 2011.

[5] S. C. Jeng, S. J. Hwang, J. S. Homg, and K. R. Lin, "Electrically switchable liquid crystal Fresnel lens using UV-modified alignment film," Opt. Exp., vol. 18, no. 25, pp. 26325-26331, 2010.

[6] H. Jashnsaz, E. Mohajerani, H. Nemati, S. H. Razavi, and I. A Alidokht, "Electrically switchable holographic liquid crystal/polymer Fresnel lens using a Michelson interferometer," Appl. Opt., vol. 50, no. 17, pp. 2701-2707, 2011.

[7] Y. Lou, L. Chen, C. Wang, and S. Shen, "Tunable-focus liquid crystal Fresnel zone lens based on harmonic diffraction," Appl. Phys. Lett., vol. 101, no. 22, 2012, Art. ID 221121

[8] K. M. Chen, S. Gauza, H. Xianyu, and S. T. Wu, "Sub-millisecond gray-level response time of a polymer stabilized blue-phase liquid crystal," J. Display Technol., vol. 101, pp. 49-51, 2010.

[9] G. Zhu, J. N. Li, X. W. Lin, and H. F. Wang, "Polarization-independent blue-phase liquid-crystal gratings driven by vertical electric field," $J$. Soc. Inf. Display, vol. 20, pp. 1-4, 2012.

[10] W. Cao, A. Muñoz, P. Palffy-Muhoray, and B. Taheri, "Lasing in a three-dimensional photonic crystal of the liquid crystalline blue phase II," Nature Mater., vol. 1, pp. 111-113, 2002.

[11] S. Yokoyama, S. Mashiko, H. Kikuchi, K. Uchida, and T. Nagamura, "Laser emission from a polymer-stabilized liquid-crystalline blue phase," Adv. Mater., vol. 18, p. 48, 2006.

[12] C. T. Lee, Y. Li, H. Y. Lin, and S. T. Wu, "Design of polarization-independent multi-electrode GRIN lens with a blue-phase liquid crystal," Opt. Exp., vol. 51, pp. 17402-17407, 2011.

[13] Y. H. Lin, H. S. Chen, H. C. Lin, Y. S. Tsou, H. K. Hsu, and W. Y. $\mathrm{Li}$, "Polarizer-free and fast response microlens arrays using polymerstabilized blue phase liquid crystals," Appl. Phys. Lett., vol. 96, 2010, Art. ID 113505.

[14] C. H. Lin, Y. Y. Wang, and C. W. Hsieh, "Polarization-independent and high-diffraction-efficiency Fresnel lenses based on blue phase liquid crystals," Opt. Lett., vol. 36, pp. 502-504, 2011.

[15] Y. H. Fan, H. W. Ren, and S. T. Wu, "Switchable Fresnel lens using polymer-stabilized liquid crystals," Opt. Exp., vol. 11, pp. 3080-3086, 2003.

[16] H. W. Ren, Y. H. Fan, and S. T. Wu, "Tunable Fresnel lens using nanoscale polymer-dispersed liquid crystals," Appl. Phys. Lett., vol. 83, pp. 1515-1517, 2003.

[17] Y. H. Fan, H. W. Ren, and S. T. Wu, "Electrically switchable Fresnel lens using a polymer-separated composite film," Opt. Exp., vol. 13, pp. 4141-4147, 2005.

[18] B. E. A. Saleh and M. C. Teich, Fundamentals of Photonics, 2nd ed. Hoboken, NJ, USA: Wiley Interscience.

[19] H. Nemati, E. Mohajerani, A. Moheghi, M. B. Rad, and N. H. Nataj, "A simple holographic technic for fabricating a LC/polymer switchable Fresnel lens," Euro Phys. Lett., vol. 87, 2009, Art. ID 64001.

[20] J. Yan, H. C. Cheng, S. Gauza, Y. Li, M. Jiao, L. Rao, and S. T. Wu, "Extended Kerr effect of polymer-stabilized blue-phase liquid crystals," Appl. Phys. Lett., vol. 96, 2010, Art. ID 071105.

[21] V. Vorflusev and S. Kumar, "Phase-separated composite films for liquid crystal displays," Science, vol. 283, pp. 1903-1905, 1999.

[22] A. Mukhopadhyay, C. L. Caylor, and B. M. Law, "Evidence for dipole surface orientational order at critical interfaces," Phys. Rev. E, vol. 61, no. 2, pp. 1036-1039, 2000.

[23] F. Castles, F. V. Day, S. M. Morris, D. H. Ko, D. J. Gardiner, M. M. Qasim, S. Nosheen, P. J. W. Hands, S. S. Choi, R. H. Friend, and H. J. Coles, "Blue-phase templated fabrication of three-dimensional nanostructures for photonic applications," Nat. Mater., vol. 11, no. 7, pp. 599-603, 2012

[24] S. Y. Lu and L. C. Chien, "Electrically switched color with polymerstabilized blue-phase liquid crystals," Opt. Lett., vol. 35, pp. 562-564, 2010.

[25] Y. Song, S. B. Ni, J. Tan, E. W. Zhong, S. Y. Liu, and J. G. Lu, "A shifting holographic fabrication for switchable LC/polymer Fresnel lens," in SID Symp. Dig., 2012, vol. 53, pp. 718-720. 


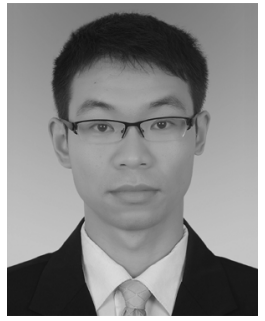

Jian Tan received the B.S. degree in Information Engineering from Shanghai Jiao Tong University in 2011.

He is currently working toward the M.S degree at the Department of of Electronic Engineering and $\mathrm{Na}$ tional Engineering Lab for TFT-LCD Materials and Technologies in Shanghai Jiao Tong University since 2011, Shanghai, China. His current research interests are 3D display technology, liquid crystal lens, blue phase liquid crystal.

Yue Song, photograph and biography not available at the time of publication

Ji-Liang Zhu, photograph and biography not available at the time of publication.

Shui-Bin Ni, photograph and biography not available at the time of publication.

Yi-Jun Wang, photograph and biography not available at the time of publication.

Xiao-Yang Sun, photograph and biography not available at the time of publication.

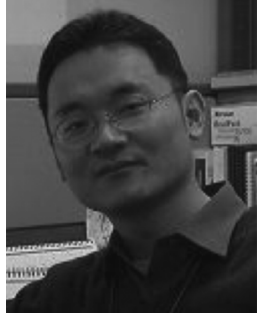

Jian-Gang Lu received the Ph.D. degree from the College of Information Science and Engineering, Zhejiang University, Hangzhou China, in 2003. He worked on LC displays with the Next-Generation LCD Research Center, LCD business, Samsung Electronics, from 2003 to 2009. Since 2009, he has been with the National Engineering Laboratory of TFT-LCD Materials and Technologies, Shanghai Jiao Tong University, Shanghai, China, as an Associate Professor. His research interest includes liquid display mode, and 3D display. crystal material, polymer material, liquid crystal

Bo-Ru Yang, photograph and biography not available at the time of publication.

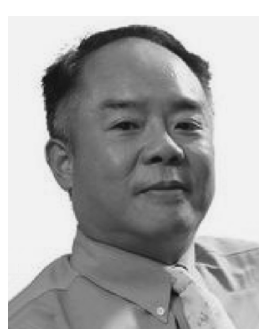

Han-Ping D. Shieh received the B.S. degree from National Taiwan University in 1975 and Ph.D. in electrical and computer engineering from Carnegie Mellon University, Pittsburgh, PA, U.S.A. in 1987.

$\mathrm{He}$ joined National Chiao Tung University (NCTU) in Hsinchu, Taiwan as a professor at Institute of Opto-Electronic Engineering and $\mathrm{Mi}$ croelectronics and Information Research Center (MIRC) in 1992 after as a Research Staff Member at IBM TJ Watson Research Center, Yorktown Heights, NY, USA since 1988. He was an Associate Director, MIRC, NCTU. He founded and served as the Director, Display Institute at NCTU in 2003, the first such kind of graduate academic institute in the world dedicated for display education and research. He was the Dean, College of Electrical and Computer Engineering, NCTU (2006-2010) and AU Optronics Chair Professor. He is now an NCTU Chair Professor and a NCTU senior vice President. He is also holding an appointment as a Chang Jiang Scholar at Shanghai Jiao Tong University since 2010

Dr. Shieh is a fellow of IEEE, OSA and SID (Society for Information Display). 